\title{
El pensamiento complejo como estrategia didáctica en las Ciencias Biológicas
}

Francisco Javier Acosta Collazo

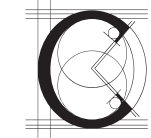

omo educadores en la sociedad del conocimiento, la realidad debería ser percibida por los estudiantes como una integración interconectada de fenómenos, eventos y consecuencias que son difíciles de ser previsibles, es decir, se presentan incertidumbres. En la docencia de las ciencias experimentales, en general, se tiende a reducir los acontecimientos o fenómenos de los sistemas naturales mediante un pensamiento lineal y fragmentado.

\section{Conocimiento pertinente}

El aumento impresionante en la información y conocimiento relacionado con las Ciencias Biológicas (biología molecular, terapia génica, cambio climático, sistemas microbiológicos, etcétera) y la inclusión de las TIC como parte de los ambientes de aprendizaje, representan grandes retos en la educación y metodologías centradas en el aprendizaje. Martínez (2012), en un interesante análisis sobre el futuro de la escuela, señala que debemos intentar comprender el lugar que ocupan los nuevos medios de comunicación de la era digital en las nuevas generaciones de jóvenes, lo cual no debe sobrevalorarse ni subestimarse; posteriormente destaca la importancia de reorientar la educación, de manera que se vuelva una opción más interesante y atractiva para los jóvenes.

Por otra parte, siguiendo las orientaciones pedagógicas internacionales para la educación del futuro, Delors (1996) señala que la educación a lo largo de la vida tiene sustento en cuatro pilares del conocimiento', los cuales han sido considerados en la

1 Los cuatro aprendizajes fundamentales que denomina pilares del conocimiento son: aprender a conocer para adquirir los instrumentos de la comprensión; aprender a hacer para poder influir sobre el propio entorno; aprender a vivir juntos para participar y cooperar con los demás en todas las actividades humanas; y aprender a ser para alcanzar la plenitud siendo artífices del propio destino. Por lo tanto, la prioridad no es sólo el conocimiento. 


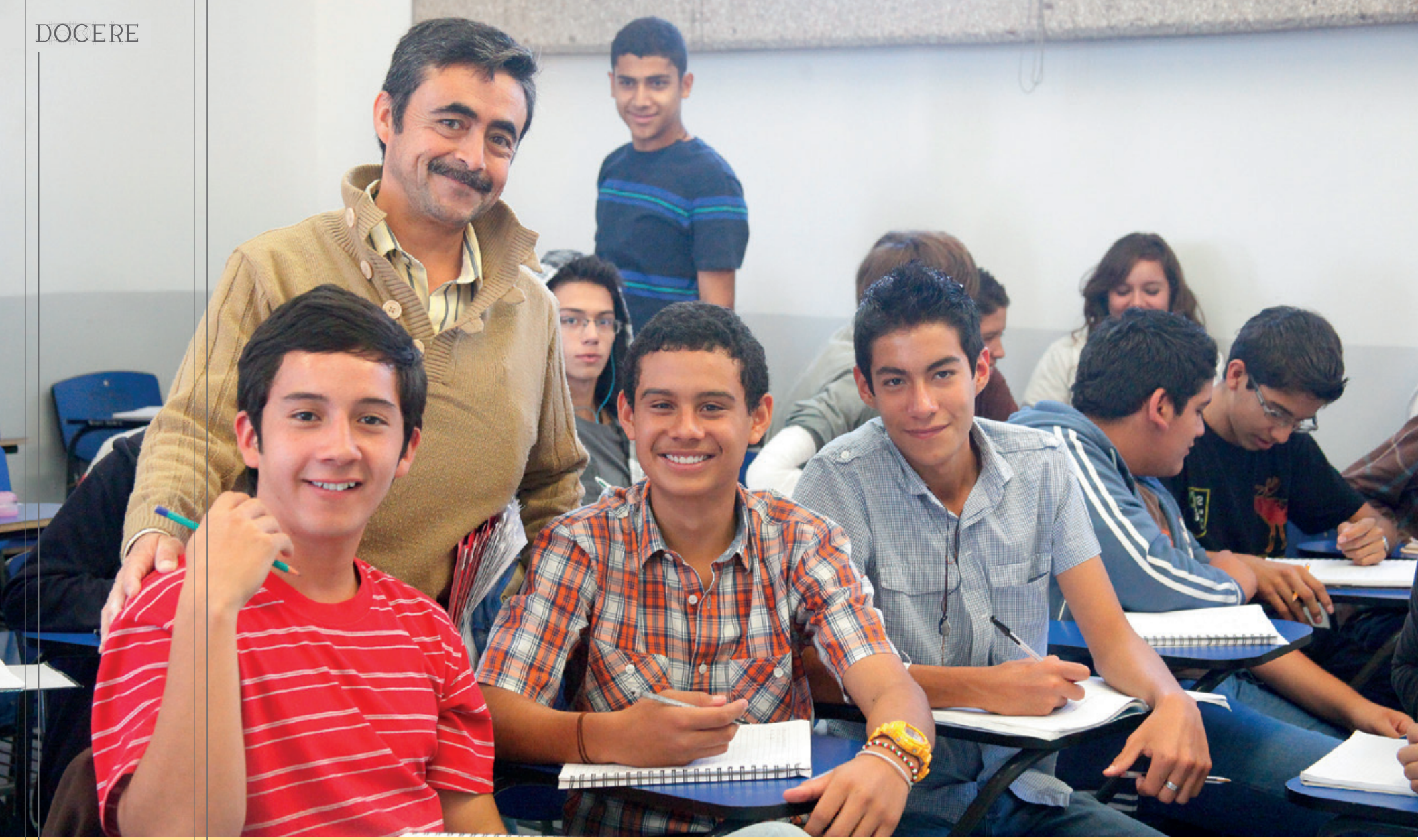

educación formal; posteriormente, la UNESCO solicita a Edgar Morín ${ }^{2}$ exponer los problemas centrales para la educación del siglo XXI publicando el texto denominado "Los siete saberes necesarios a la educación del futuro", en donde el segundo saber se enmarca en los principios de un conocimiento pertinente que debe enfrentar la complejidad ${ }^{3}$ ante saberes desunidos, parcializados y realidades multidimensionales con problemas globales. Morín (1999) clasifica en siete capítulos los saberes fundamentales para la educación del futuro. ${ }^{4}$

En el contexto institucional, la UAA aprueba el Modelo Educativo Institucional (2006), en donde se señala la diferencia entre el proceso de aprendizaje que considera el aprendizaje como finalidad de la enseñanza y el proceso de enseñanza, que promueve el desarrollo de la capacidad para procesar la información, interpretarla, darle uso, así como aprender a convivir en condiciones de incertidumbre, lo cual se vincula con el aumento significativo de la información.

Posteriormente, la Reforma Integral de la Educación Media Superior (RIEMS, 2008), define las orientaciones necesarias para generar el currículo por Competencias del Bachillerato de la UAA (2011), en donde se incluye un conjunto de competencias que pretenden facilitar a

2 Presidente de la Agencia Europea de la Cultura unEsco y del Instituto Internacional para el Pensamiento Complejo (IIPC): http://www.complejidad.org/.

3 Complejidad no equivale a complicación o confusión, la complicación se puede resolver en forma ordenada y no considera las incertidumbres.

4 Morín sostiene que hay siete saberes que la educación debe tratar: un saber que supere las cegueras del conocimiento; un saber que funde los principios de un conocimiento pertinente; un saber que enseñe la identidad terrenal; un saber que permita enfrentar las incertidumbres; un saber que enseñe la comprensión; un saber que enseñe la condición humana y un saber que enseñe la ética del género humano. 
los jóvenes aprender a aprender, tener una visión del mundo en toda su complejidad.

\section{¿Qué es lo que no se ve?}

Para el caso de las ciencias experimentales en el Centro de Educación Media (CEM), la primera competencia del ámbito conceptual señala: "Interpreta de manera crítica y reflexiva el mundo natural a través de la comprensión de redes de conceptos así como del acercamiento al método científico" (UAA, 2011: 63). En relación con la complejidad y en un acercamiento a los significados de conceptos vinculados con el saber ambiental en jóvenes del bachillerato de la UAA, los resultados de percepción relacionados con el concepto de complejidad se vinculan con dificultad en un mayor porcentaje (Acosta, 2006: 41-42), lo cual indica que la concepción en los estudiantes deberá ser reconstruida. Al abordar un concepto desde la complejidad, se deben considerar las diversas variables en los componentes con relaciones dinámicas e interdependientes; entonces para lograr la comprensión de algún fenómeno, se pueden visualizar las cualidades o propiedades que se producen por la organización del sistema, es decir, las propiedades son más que la suma de sus componentes.

Un pensamiento complejo no significa un pensamiento completo, como bien lo describen Morín, Roger y Motta (2003: 64) en el apartado denominado "Características del pensamiento complejo". Para efectos de esta propuesta, nos limitaremos a definir el pensamiento complejo ${ }^{5}$ como un enfoque integrador en el que el todo integra las partes y las partes sólo presentan al todo, además de su enfoque aplicado al aprendizaje de la Biología.

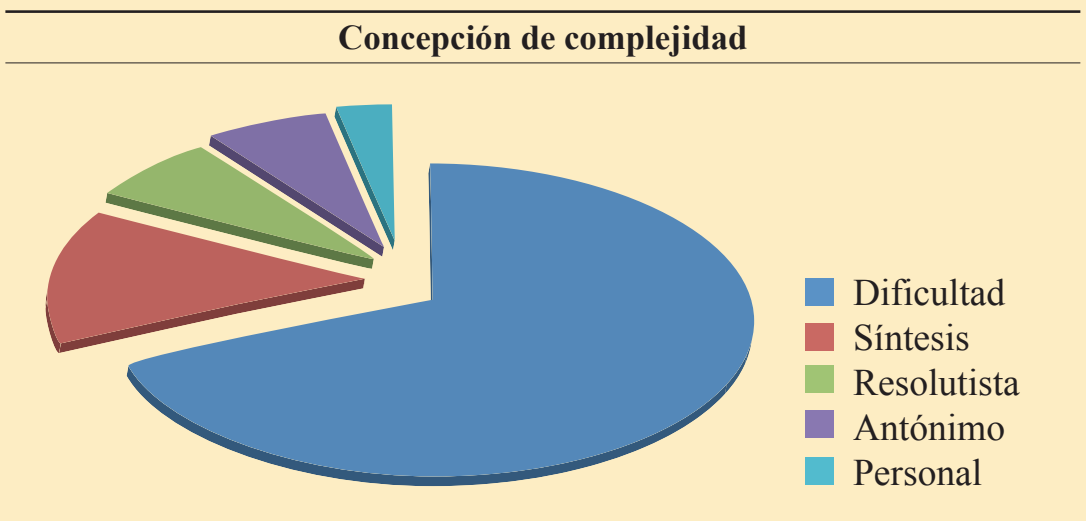

Fuente: Acosta (2006).

Por ejemplo, el metabolismo de una célula implica miles de reacciones químicas que se producen en forma dinámica y simultánea para su mantenimiento, sintetizar estructuras básicas como las proteínas, eliminar productos de desecho, comunicarse con su entorno, etc., si

5 Malinowski (2012) presenta una variedad de ejemplos para clarificar el pensamiento complejo en amena vídeo-conferencia denominada: Introducción al pensamiento complejo: www.multiviversidadreal.edu.mx/conferencia-introducción-pensamiento-complejo.html. 
se analiza solamente el tema de estructura celular, podemos "no ver" la interacción de las diferentes partes de los componentes.

\section{Complejidad en las Ciencias Biológicas}

En este acercamiento del concepto de complejidad, los sistemas biológicos presentan ejemplos claros de las propiedades en los sistemas, lo cual se conoce como propiedades emergentes que tiene antecedentes y fundamento en la visión sistémica de la vida o pensamiento sistémico, así el texto La trama de la vida, de F. Capra (1996) es una excelente obra que valora interrelaciones e interdependencias de los fenómenos y sistemas vivos.

También el ecólogo J.E. Lovelok valora las interacciones y presenta un estudio sobre la composición de los gases de la atmósfera y su relación con los organismos unicelulares, que forman parte de la flora intestinal de otros organismos, como los rumiantes, los cuales generan cantidades importantes de metano ${ }^{6}$. Entonces, los organismos que podemos interpretar como insignificantes, en realidad forman parte de la dinámica de la biosfera como un todo.

Con esta perspectiva, se identifica una gran variedad de temas como el genoma humano, cambio climático, el micromundo celular, el ADN y el flujo de información, las enfermedades como producto de la contaminación, y hasta la vida misma podría ser considerada como una propiedad emergente. Éstos y otros temas se pueden transformar a experiencias didácticas desde la perspectiva del pensamiento complejo.

\section{Estrategia de aprendizaje}

Actualmente en el curso de Ecología y Desarrollo Sustentable del CEM se promueven estrategias de construcción de estructuras mentales en los estudiantes mediante estrategias de aprendizaje como el mapa mental o red de conceptos como producto del tema biosfera, con el propósito de organizar y visualizar los conocimientos o saberes desde una perspectiva más amplia. Los mapas mentales como herramienta estratégica se fundamentan en los nuevos aportes de la Neurofisiología, que identifica el proceso de la información en los hemisferios cerebrales. "Es una manera de generar, registrar, organizar y asociar ideas y tal como las procesa el cerebro humano, para plasmarlas en un papel" (Zambrano y Steiner, 2000: 75). En este caso se pretende valorar las interacciones que tiene todo ser viviente con su entorno natural; microorganismos, plantas y animales están interconectados, unidos por vínculos que conforman un equilibrio o un todo.?

A grandes rasgos, la primera etapa de la estrategia sugiere, en un primer momento, realizar un diagnóstico de saberes en relación con el tema de la biosfera, mediante una lluvia de ideas en una sesión grupal. La segunda etapa plantea visualizar el vídeo "Home", disponible en YouTube, ${ }^{8}$ y a partir del vídeo, los estudiantes en equipo elaboran por escrito un mapa mental que podrá ser coevaluado. El profesor hará

6 Gas que forma parte de los gases de invernadero en la atmósfera.

7 Nada se basta a sí mismo.

8 HomeprojectES. (Mayo 19 del 2009), Home (ES), [Archivo de video]. Obtenido de: http:// www.youtube.com/watch?v=SWRHxh6XepM. 
énfasis en las interacciones de manera que se visualicen las propiedades del sistema. Como una tercera etapa, se propone que los estudiantes en forma individual elaboren un mapa mental con las propiedades del sistema y envíen un documento electrónico al profesor ${ }^{9}$ con los términos establecidos durante la primera sesión finalmente, para la evaluación de los aprendizajes, se propone utilizar la rúbrica diseñada como parte de la estrategia.

\section{Algunas conclusiones}

Como parte de los cambios requeridos en la sociedad del conocimiento, están los nuevos enfoques centrados en el aprendizaje, los cuales marcan una diferencia en relación con el modelo pasivo de enseñanza y de transmisión de conocimientos, a un modelo en el que el estudiante, es el gestor del conocimiento y la información. El pensamiento crítico y sistémico es vigente y se fundamenta con las competencias desde la perspectiva de la complejidad.

Las Ciencias Biológicas, al igual que las experimentales, presentan contextos en donde se pueden identificar las propiedades emergentes que presentan la perspectiva de complejidad y que son un área de oportunidad para la aplicación de estrategias de aprendizaje.

\section{Fuentes de consulta}

Acosta, F.J. (2006). Construcción del saber ambiental en alumnos del bachillerato de la UAA. Tesis de maestría, U. de G., México.

Capra, F. (1996). La trama de la vida. Una nueva perspectiva de los seres vivos. Barcelona: Anagrama.

Delors. J. (Coord.). (1996). La educación encierra un tesoro. Compendio del Informe a la UNESCO de la Comisión Internacional sobre Educación para el Siglo XXI. UNESCO. Recuperado el 15 de agosto de 2013 en: http://unesdoc .unesco.org/images/0010/001095/109590so.pdf.

Martínez, F. (2012). La escuela y el futuro. Alegato por la esperanza. México: UAA.

Morin E. (1999). Los siete saberes necesarios para la educación del futuro. Organización de las Naciones Unidas para la Educación, la Ciencia y la Cultura, Francia. UNESCO.

Morín, E., Roger, E. y Motta, R. (2003). Educar en la era planetaria. Barcelona: Gedisa.

RIEMS. (2008). Acuerdo Secretarial 442, Diario Oficial, México. Recuperado el 15 de agosto de 2012 en: http://www.reforma-iems. sems.gob.mx/wb/riems/acuerdos_secretariales.

Zambrano, J. y Steiner, A. (2000). Mapas mentales. Agenda para el éxito. México: Alfadil.

UAA. (2011). Modelo Educativo Institucional (MEI). Recuperado el 27 de julio de 2011 en: http://www.uaa.mx/direcciones/dgdp/defaa/ descargas/modelo_educativo_folleto_mayo.pdf.

UAA. Bachillerato General, Curriculum por Competencias 2011. México: UAA.

9 Generalmente es la plataforma educativa. 